\title{
Modeling and Simulation on Revolving Movement of Log for Veneer Lathe
}

\author{
Xiong Guang Ming ${ }^{1}$ Guo Li Jun ${ }^{1}$ \\ ${ }^{1}$ Dept. of Aeronautics, Xiamen University, Xiamen, China \\ xgm@xmu.edu.cn
}

\begin{abstract}
.
The mathematical variable-speed feeding model of cutter was established., the changing law between the cutter feeding speed and the diameter of the log was deduced, the calculation method of the peeling time was also derived, The feeding speed must be changed in accordance with the law, which makes the lathe work well. Based on the founded moving track model of invariable-speed peeling and deduced accurate calculating formula of invariable-speed peeling, the approximate calculating formula of peeling time was also derived. This article generates a simulation with the help of MATLAB software. the results of study will provide a guide for the control system design.
\end{abstract}

Keywords: Veneer lathe. Revolving movement. Modeling.

\section{Introduction}

The veneer lathe is a main machine in plywood production, it is used to peel the log into veneer, in order to raise the utilization ratio of the log, veneer lathe was widely used at present. It is the main problem that the precision of the veneer and the uniformity of the thickness are acquired. In rotary cutting process, the movement of the knife is the most crucial, including the feeding-speed, the rotary cutting time, Therefore, establishing the cutting model, studying on the changing law between the serial movement parameters of the cutter and the log can provide the basic theory for design of system.

The mathematical model of veneer lathe is shown in figure 1. 


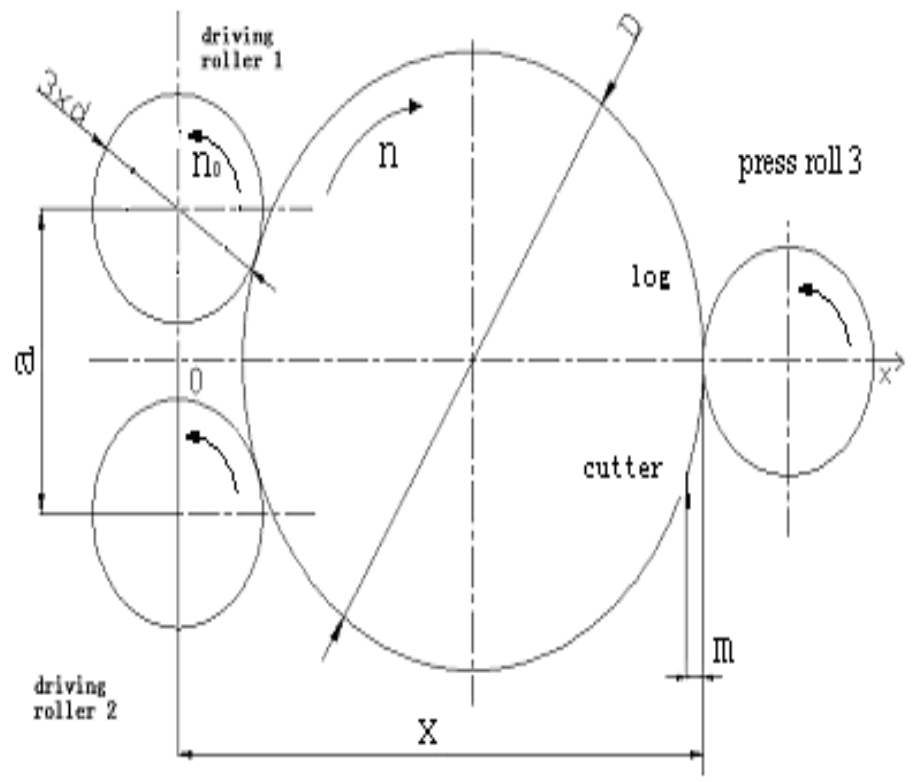

Fig.1 The system sketch of the veneer lathe

Driving roller 1, 2 and press roll 3 contra rotate. The knife fixed on the press roll 3 is fed at the speed of $\mathrm{v}(\mathrm{m} / \mathrm{s})$. When the rotary speed $n_{0}$ of the driving roller 1 , 2 and the press roll 3 keep on constant, the rotary speed of the log is $n(r / m)$ and the rotary speed $\mathrm{n}$ of the $\log$ will be changed. Supposing that there is no relative sliding among the driving rollers, press roll and the log, then the tangent linear velocity of the log is constant. So in order to peel veneer with the same thickness $\mathrm{m}$, in rotary cutting process, it requires the feeding speed $\mathrm{v}$ of the knife must be changed continuously according to the law, which makes the lathe work well.

\section{Cutter with Variable-speed Model}

Here: d: the diameter of driving roller 1,2 and driving roller $3 ; n_{0}$ : rotating speed per minute (rpm); 
D: the diameter of the round log;m: peeling thickness (mm);a: the central distance of the driving roller1, $2(\mathrm{~mm})$; v: feed speed $(\mathrm{m} / \mathrm{s})$;

The tangent linear velocity of the round log is equal to that of the driving roller, thus:

$$
D \times n=d \times n_{0}
$$

In the veneer lathe peeling process, when the rotation speed $n_{0}(\mathrm{rpm})$ of the driving roller keeps on constant; the rotation speed of the round log is increasing while the diameter of the round log is decreasing.

When the veneer lathe peels the round log, its peeling knife edge is in the symmetrical center plane of the driving roller1, 2, the horizontal coordinates of the peeling knife edge is:

$$
x=\frac{D}{2}+\frac{1}{2} \sqrt{(D+d)^{2}-a^{2}}
$$

The first derivative of the equation (2) with respected to the time $t$; so, the horizontal moving speed of the veneer lathe will be got:

$$
V=\frac{d x}{d t}=\frac{1}{2} \times\left[1+\frac{D+d}{\sqrt{(d+D)^{2}-a^{2}}}\right] \times \frac{d D}{d t}
$$

Substitute (1) and $\frac{d D}{d t}=-2 \times m \times n$ into (3), thus

$$
V=\frac{d x}{d t}=-\left[1+\frac{D+d}{\sqrt{(D+d)^{2}-a^{2}}}\right] \times m \times \frac{d \times n_{0}}{D}
$$

A negative sign of (4) indicates that the direction of $v$ is in the opposite direction of the coordinate axis.To guarantee the same thickness of the veneer, the cutting variable-speed $\mathrm{v}$ should be equation (4).Choose: $a=97 \mathrm{~mm} \quad, \quad m=0.6-3 \mathrm{~mm} \quad, \quad n_{0}=74 \mathrm{rpm} \quad, \quad D_{\max }=380 \mathrm{~mm}$, $D_{\text {min }}=25 \mathrm{~mm}, d=96 \mathrm{~mm}$ 


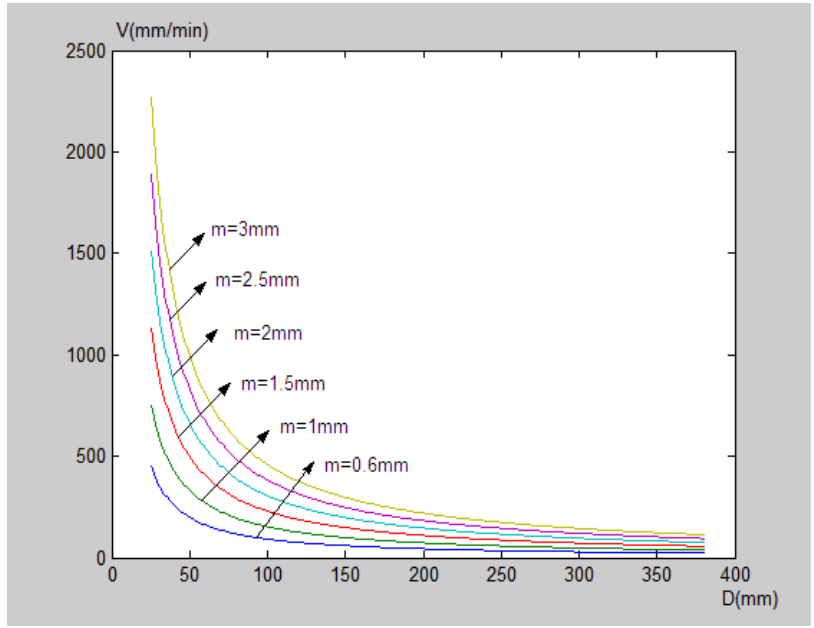

Fig.2 The changing curve of the blades feeding speed $v$ and the diameter $\mathrm{D}$ of the $\log$

Substitute the above parameter into (4), emulate with MATLAB, thus we can get the changing curve of the cutting variable-speed $\mathrm{v}$ change with the diameter $\mathrm{D}$ of the round log. Its diagram is shown in figure 2.

From figure2 we can see that when $\mathrm{d}$, a, $n_{0}$, $\mathrm{m}$ is fixed, the knife feeding speed $\mathrm{v}$ changes with the diameter of the $\log \mathrm{D}$. In the veneer lathe peeling process, $\mathrm{D}$ became smaller and smaller, while $\mathrm{v}$ became bigger and bigger. The bigger $\mathrm{m}$ is, the quicker the knife feeding speed is.

The precise calculation of peeling time $t$.

Because:

$$
\frac{d D}{d t}=-2 \times m \times n
$$

Integral equation (5), thus:

$$
\int_{D_{0}}^{D} D \times d D=\int_{0}^{t}-2 \times m \times n_{0} \times d \times d t
$$




$$
t=\frac{D_{0}^{2}-D^{2}}{4 \times m \times n_{0} \times d}
$$

Equation (4)、(7), if use the same above numeric value, through the emulation of MATLAB. The change curve of knife feeding speed change with the time, its diagram is shown in figure 3.

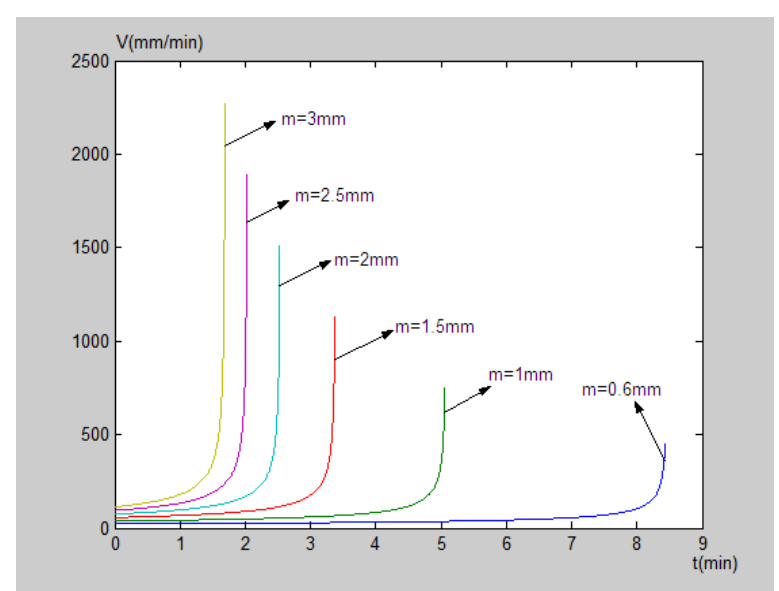

Fig.3 The changing curve of the blades feeding speed and the time

From figure3 we can see that when $\mathrm{d} 、 \mathrm{a} 、 n_{0} 、 \mathrm{~m}$ is fixed, the knife feeding speed $\mathrm{v}$ became bigger along with time $\mathrm{t}$. In the veneer lathe peeling process, thickness $\mathrm{m}$ became bigger, the knife feeding speed is quickly incremented and peeling time decreases.

\section{Calculation and simulation of the pitch angle}

In rotary cutting process, the curvature was increased with the diameter of the log decreased. In feeding process, if the interface between the back of the knife and the log is constant, the pitch angle $\varphi$ of the knife should be changed.

It is shown in figure 4.Here, $\mathrm{H}$ is the contact height between the knife and the log, $\mathrm{r}$ is the radius of the $\log , \mathrm{O}$ is the rotary center of the log, $\mathrm{A}$ is the edge of the 
cutter, $\mathrm{AB}$ is the contact line between the back of the cutter and the log, $\varphi$ is the pitch angle.

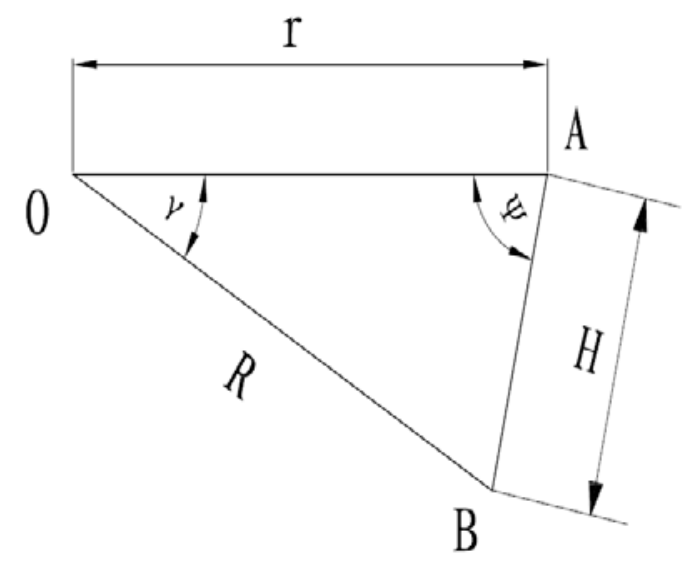

Fig.4 The triangle among the pitch angle and other parameters

In $\triangle O A B$, according to the Cosine Theorem, the equation (8) will be got:

$$
\cos \varphi=\frac{r^{2}+H^{2}-R^{2}}{2 r H}
$$

According to the Sine Theorem again, $R=H \frac{\sin \varphi}{\sin \gamma}$, Substitute into (8), The unary quadric equation about $\cos \varphi$ is solved, The equation (9) will be got:

$$
\cos \varphi=\frac{r \sin ^{2} \gamma-\sqrt{\left(1-\sin ^{2} \gamma\right)\left(H^{2}-r^{2} \sin ^{2} \gamma\right)}}{H}
$$

Because the value of the height $\mathrm{H}$ which relative to the value of the radius $\mathrm{r}$ is very small, according to the bowstring relationship, we will obtain:

$$
\begin{gathered}
\gamma=2 \arcsin \frac{H}{D} \\
\cos \varphi=\frac{D \sin ^{2} \gamma-\sqrt{\left(1-\sin ^{2} \gamma\right)\left(4 H^{2}-D^{2} \sin ^{2} \gamma\right)}}{2 H}
\end{gathered}
$$


The equation (10) is substituted into (11), the pitch angle $\varphi$ will be solved, the mathematic relationship among $\varphi, \mathrm{H}$ and $\mathrm{D}$ will be obtained. $\mathrm{H}$ can be controlled artificially and commonly $\mathrm{H} \leq 4 \mathrm{~mm}$. Choosing : $\mathrm{H}=4 \mathrm{~mm}, 3 \mathrm{~mm}, 2 \mathrm{~mm}$, $1 \mathrm{~mm}$, the change of the pitch angle was simulated with MATLAB software and its curve was shown in Figure 5.

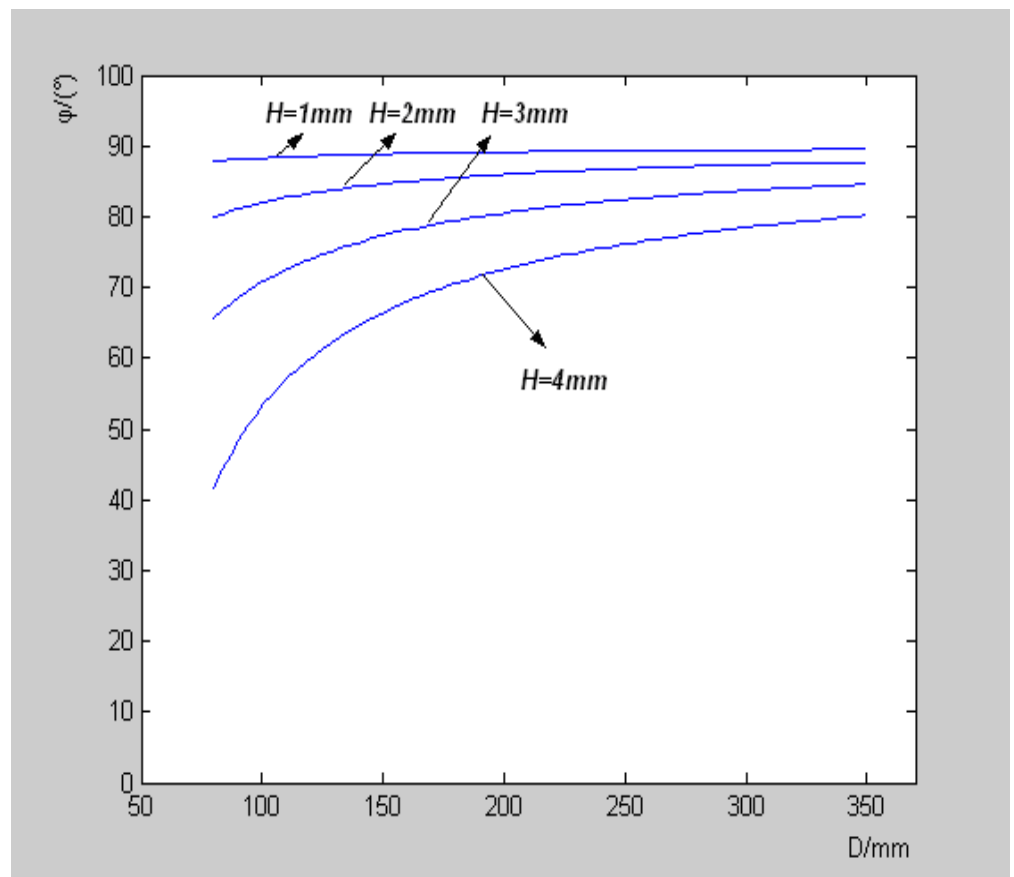

Fig. 5 The changing curve between the pitch angle and the diameter of the log

The Figure 5 shows that when the height $\mathrm{H}$ was constant, the pitch angle $\varphi$ was related with the diameter $\mathrm{D}$ of the log. In rotary cutting process, the pitch angle $\varphi$ was decreased with the diameter of the log decreased, which was faster and faster. The bigger the chosen height $\mathrm{H}$ was, the faster the speed of the pitch angle $\varphi$ changed. In addition, by simulation curve, it was shown that when the diameter of the log was bigger than $200 \mathrm{~mm}$, the change of the pitch angle was a line, when the diameter of log was smaller than $200 \mathrm{~mm}$, the change of the pitch angle was a curve and the decrease of the pitch angle was accelerated with the decrease of the diameter of the log. 


\section{Conclusion}

In order to achieve the optimization of the rotary cutting system, when the knife was fed at variable speed it must be rotated. The rotation of the knife means the change of the pitch angle. The pitch angle was decreased with the diameter of the log decreased, which was faster and faster according to the curve. The bigger the chosen height $\mathrm{H}$ was, the faster the speed of the pitch angle changed. The changing law of the pitch angle of the knife provided the theory method for control knife of the veneer lathe with high precision. The mathematical model of the variable-speed feeding of the knife can be used to guide the control system design for veneer lathe.

\section{References}

[1] W. WEI, P. Y. XI. The research and design for timber lathe and its mechanism. Machine Design and Research. 2004, 20 (3): 19-20.

[2] T. PAN, S. CHEN, X. Y. CHEN,J. H. WANG. Variable-speed feeding mechanism and control system designed of log-core veneer lathe, Modular Machine Tool \& Automatic Manufacturing Technique. 2003, 2: 13-64.

[3] X. Lu, G. M. Xiong,G. Q. Hu. Design and research on automatic control system for hydraulic log-core veneer lathe. 3rd China-Japan Conference on Mechatronics. FuZhou. 2006, pp. 120-123.

[4] X. Lu, G. M. Xiong,G. Q. Hu. Study on the model both cutting variablespeed of the log-core veneer lathe and moving locus model with constant-speed peeling. Forestry Machinery \& Woodworking Equipment. 2006, 34 (1): 12-15.

[5] J. L. Wang, P. X. Cao. Variation of working clearance angle during rotary veneer cutting. China Wood-Based Panels. 2004, 11 (7): 11-13. 